\title{
Aesthetics for Communicative Visualization: a Brief Review
}

\author{
Maryam Riahi* \\ North Carolina State University
}

\author{
Benjamin Watson ${ }^{\dagger}$ \\ North Carolina State University
}

- had at least 10 citations

- were published in the previous 20 years

- clearly addressed our topic

In addition, our introductory sections include some additional, largely historical publications that set context.

\section{Understanding Aesthetics}

According to Merriam-Webster [51], aesthetics is "a branch of philosophy dealing with the nature of beauty, art, and taste and with the creation and appreciation of beauty." The term was first coined by German philosopher Alexander Baumgarten, and was later popularized by Kant, meaning the "science which treats of the conditions of sensuous perception" [23]. More recently, Palmer et al. [58] define aesthetics as "the study of those mental processes that underlie disinterested evaluative experiences that are anchored at the positive end by feelings that would accompany verbal expressions such as 'Oh wow! That's wonderful! I love it!' and at the negative end by 'Oh yuck! That's awful! I hate it!"'

Applied researchers have a much more practical perspective. Tractisky et al. [75] use the everyday sense, "the beauty or the pleasing appearance of things." Cawthon and Moere [13] are more experiential: "An anesthetic is used to dull or deaden, causing sleepiness and numbness. In contrast, aesthetic is seen as something that enlivens or invigorates both body and mind, awakening the senses."

Is beauty in the eye of the beholder? This is an age-old debate [71]. The objective theory (beauty is attached to the viewed object) dominated until the middle ages, with classical philosophers like Aristotle, Vitruvius, and Plato believing that beauty dwells in objective properties such as symmetry, order, proportion, and harmony [4, 15, 82]. Later, Kant [38] and Hume [33] argued that beauty is subjective. Today, theorists $[54,70]$ hold the middle ground, advocating that beauty is an interaction between the object and the viewer. Experiential evidence exists supporting both perspectives. For instance, Langlois found [42] that facial attractiveness is universal across culture and age — even among infants [43]. Meanwhile, various cultures, genders, ages, and even contexts make different color judgements [58]. Jacobson et al. [35] believed that group models of aesthetic judgment are misleading.

\subsection{Models of Aesthetic Experience}

Berlyne [7] classifies aesthetic variables as psychophysical, collative, and ecological. Psychophysical variables can heighten or moderate emotional arousal and include color, intensity, size, and form. Collative variables are evaluative; with novelty, expectations, conflict, complexity, instability, ambiguity and multiple-meaning increasing arousal; while familiarity, dominance, grouping and patterning reduce it. Ecological variables are learned associations from the viewer's life that may arouse or calm. To experience aesthetic pleasure, arousal must be moderate: neither too low nor too high.

Much more recently, Leder et al. $[45,46]$ proposed a model integrating existing theories and results (Fig. 1). In it, aesthetic experience starts at a low, subconscious level and moves to a high, conscious level; or in Kahneman's terminology [37], begins in the "fast" mind, and moves to the "slow" mind. The experience begins with a rapid perception of a visual's low-level features. Next, integrating subconscious memory, the viewer assesses familiarity, 


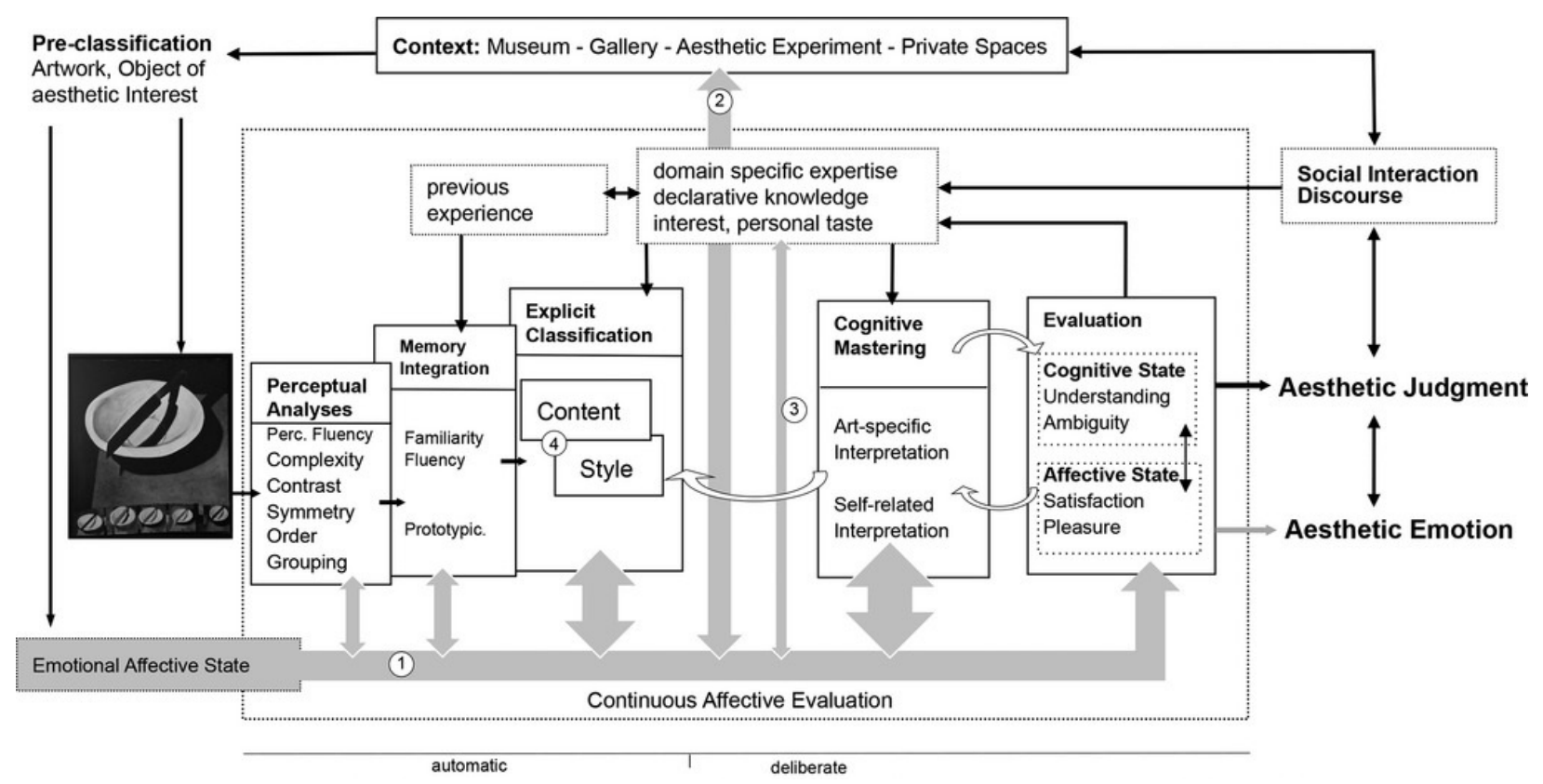

Figure 1: A Theoretical Model of Aesthetic Experience (from Leder and Nadal [46]).

prototypicality ("averageness," or how well the visual represents a category), and processing fluency (ease of understanding). During explicit classification, the viewer recognizes content and classifies style. In the last stages, cognitive mastery and evaluation seek understanding and pleasure, with success delivering a rewarding experience. Emotion is continuously present, affecting all stages of the experience. The experience can be modulated by factors such as knowledge, expertise, personal interest, social processes, and mood $[24,34]$. In over a decade, few studies have tried to verify Leder et al.'s model experimentally, with Bölte et al. [8] being one exception.

\subsection{Aesthetic Axes and Cognition}

As these models describe, beauty is a complex construct formed by the viewer from several components, which have been organized along certain axes by researchers. For example, Lavie and Tractinsky [44] developed a survey assessing website aesthetics using these components, and with factor analyses found evidence that the aesthetic components could be organized along two dimensions: classical and expressive. The classical dimension responds to clean, clear, symmetric and pleasant design; while the expressive dimension corresponds to original, creative, fascinating, and sophisticated design. Moshagen and Thielsch [55] found four dimensions: colorfulness, simplicity, diversity, and craftsmanship; with simplicity and diversity highly correlated to Lavie and Tractinsky's classical and expressive axes. These two dimensions may seem opposites on the same axis, instead of independent axes. However, in accordance with Fechner's quote "the right combination of diversity in unity" [20], viewers judge classical and expressive axes independently. While studying participants' responses to art objects, Baltissen and Ostermann [3] found two other axes: emotion, comprising responses to warm, arousing, and dominant visuals; and cognitive, made up of pleasing, interesting, familiar, and simple responses.

Several researchers have argued for the importance of considered, higher-level cognition in aesthetic experience, despite the strong evidence for rapid, subconscious response $[19,47,48,79,85]$. Gestalt psychologists like Arnheim [2] emphasized the role of cognition in obtaining meaning from visuals. Martindale [49] proposed that the pleasure induced by a visual is a function of the cognitive units that decode it. Later, Martindale et al. [50] demonstrated that Berlyne's collative variables are not as important as higher-level semantics in determining aesthetic preference. While Kahneman [37] has famously argued for two cognitive systems - one fast and low-level, the other slow, and high-level; Don Norman [56] proposed three levels to aesthetic interaction: a fast, visceral level; a slower behavioral level based on expectations; and a slowest reflective level in which people attach meaning to previous experience. Neuroaesthetics research has found evidence supporting such contemplative aesthetic reactions $[14,29]$.

\subsection{Aesthetic Components and Their Measurement}

Space limitations do not allow us to describe all of the proposed components of appealing aesthetics, but we describe some of the most widely examined and complex here, ordered roughly from fast, low-level to slow, high-level cognition.

Colorfulness is widely preferred by viewers, but some colors and combinations are exceptions, with preferences depending on culture and individual experience. [57,58,72]. Complexity is another component [52], with viewers preferring it in moderation: neither too little, nor too much [78]. Symmetry is one of the Gestalt laws, and may be the most important of the spatial aesthetic components [6,44], with balance a less strict version of it. Viewers also prefer symmetry in moderation [7]. Other spatial components include the "golden section" [71], alignment, sequencing, and grouping [2].

Movement, rhythm, unity and regularity are other low-level aesthetic components that have not been well-studied [60].

Two important higher-level aesthetic components are familiarity and prototypicality ("averageness", or how well the visual represents a category) $[1,7,26,45]$. "Peak-shift" guides user attention, but study of its effects on aesthetic preference is scarce [45,65]. Study of content recognition, style classification and craftsmanship detection are still more rare [55].

Measuring the presence of these components in a visual can be challenging. The most common method is subjective surveys $[48,50,55,77]$. Similar surveys can be used to validate computational models $[18,41,67,84,86]$, including computer vision alternatives to subjective surveys. Higher-level, expertise- and memory-dependent aesthetic components such as familiarity and prototypicality are 
Table 1: The number of papers discussing each aesthetic component, by field.

\begin{tabular}{|c|c|c|c|c|}
\hline Subject Area & Visualization & $\begin{array}{c}\text { Graphic Design/ } \\
\text { Marketing }\end{array}$ & $\begin{array}{c}\text { Psychology/ } \\
\text { Neuroscience }\end{array}$ & Other \\
\hline Reference Count $*$ & 8 & 8 & 18 & 11 \\
\hline Complexity/Simplicity & 6 & 4 & 6 & 9 \\
\hline Colorfulness/Monochromatic & 3 & 1 & 6 & 5 \\
\hline Familiarity/ Novelty & 2 & 0 & 2 & 1 \\
\hline Prototypicality/ Uniqueness & 1 & 0 & 4 & 1 \\
\hline Symmetry/ Asymmetry & 2 & 4 & 1 & 0 \\
\hline Balance & 0 & 1 & 0 & 0 \\
\hline
\end{tabular}

* Ignoring dictionary, encyclopedia, glossary, arts/philosophy, and unrelated references

** Excluding the references only mentioning or just implying aesthetics

Table 2: The number of papers using each type of aesthetic measurement, by field.

\begin{tabular}{|c|c|c|c|c|}
\hline Measurement & Visualization & $\begin{array}{c}\text { Graphic Design/ } \\
\text { Marketing }\end{array}$ & $\begin{array}{c}\text { Psychology/ } \\
\text { Neuroscience }\end{array}$ & Other \\
\hline Reference Count $*$ & 14 & 8 & 10 & 8 \\
\hline Self report & 10 & 6 & 8 & 6 \\
\hline Physiological & 1 & 1 & 0 & 1 \\
\hline Behavioral & 3 & 1 & 1 & 1 \\
\hline Cognition & 0 & 0 & 1 & 0 \\
\hline
\end{tabular}

* Ignoring dictionary, encyclopedia, glossary, arts/philosophy, and unrelated references

** Excluding the references only mentioning or just implying aesthetics

less amenable to computational alternatives $[25,73]$. However, in measuring prototypicality, some researchers have used objective measures of average similarity [26]. The highest-level aesthetic components, such as content recognition and style classification, are the most difficult to measure - though Moshegen et al. used a survey to observe craftsmanship detection [55]. The results of aesthetic experience, such as understanding, satisfaction, emotion and attitudes are much better researched and can be measured with a range of methods, including standard subjective surveys as well as behavioral and physiological methods [21,68].

Table 1 lists the most significant aesthetic components, and the number of papers from each discipline (psychology, graphic design and visualization) that discuss each of them.

\section{AESTHETICS IN GRAPHIC DESIGN}

According to the American Institute of Graphic Arts (AIGA), "graphic designers find beauty within limitations, working towards the ultimate goal of visually communicating a message. Utilizing a language of type and imagery, graphic designers try to make every aspect of our lives defined and beautiful" [16]. Clearly, given the importance of beauty in their practice, graphic designers are concerned with aesthetics. This becomes even clearer when one considers that many of the basic elements that concern graphic designers (e.g. symmetry, color, balance, unity $[16,83]$ ) are often considered components of beauty. But why do graphic designers value beauty? Arguing against what they see as modernism's preference for function over beauty, Sagemeister and Walsh [69] argue that beauty improves function.

Historically, graphic design is a professional rather than a research discipline, and so our search found only a few peer-reviewed graphic design publications that address aesthetics - and in fact many were collaborations with researchers from other disciplines. The papers we found bring a practical perspective that sometimes does not bother with theoretical definitions. For example in their work on computational modeling of the aesthetics of logos, Zhang et al. [86] did not define aesthetics, instead simply relying on surveys of human judgement. While studying the value of art in ambient visualizations, Huh et al. [32] used semi-structured interviews to elicit aesthetic reactions. Pham [64] seeks to understand the relationship between aesthetics and basic product characteristics (e.g. shape, composition and color) so that aesthetic design might ultimately be automated. He defines aesthetics instrumentally as a description of "the characteristics of the appearance of a design," particularly those involving an emotional, non-utilitarian response. Pham does not measure aesthetics in his own work. Finally, Jacobsen and Höfel $[28,35]$ research the human aesthetic judgement of abstract geometric patterns, using psychological methods. They rely on previous psychological definitions of aesthetics, such as those from Fechner [20] and Berlyne [7]. Participants indicated aesthetic preference by sorting the patterns into "beautiful," "not beautiful," and "indifferent;" while the pattern characteristics (e.g. symmetry, complexity) that correlated with aesthetic preference were derived using mathematical regression.

The work by Zhang et al. [86] and Pham [64] suggests that automated tools could help assess the aesthetic appeal of new visuals. Both Zhang et al. [86] and Jacobsen and Höfel [28,35] offer evidence that designers can give visuals more appeal by improving certain components of aesthetics: for logos, contrast, unity and balance [86]; for abstract geometric figures, symmetry and complexity [28,35]. Jacobsen and Höfel go further, identifying a significant contrary minority that prefers less symmetry and complexity [35], and finding that aesthetic judgements - at least of abstract figures — change 


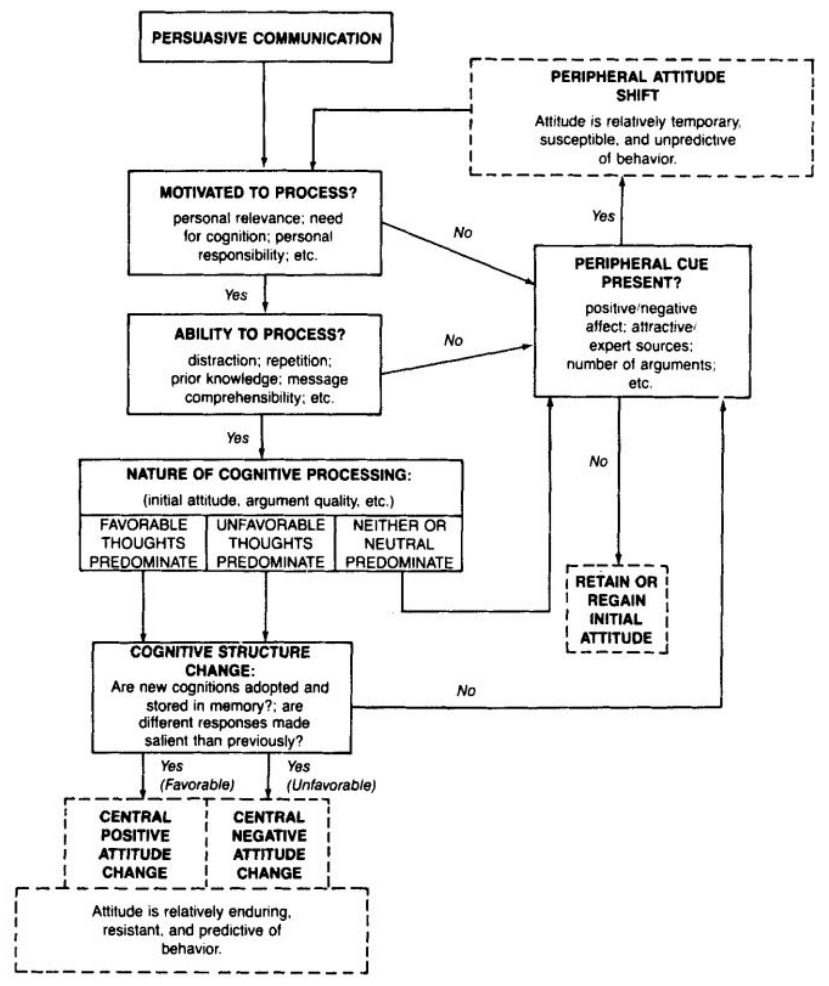

Figure 2: The Elaboration Likelihood Model (ELM), with the central route on the left, peripheral on the right. From [62].

significantly after several weeks [28]. Responses during Huh et al.'s semi-structured interviews [32] also suggest caution: at least with more complex visuals, certain aesthetic treatments may obscure or interfere with the core visual message.

\section{AESTHETICS IN MARKETING}

Marketing seeks to create and communicate offerings that have value for customers and society at large [36]. Aesthetics have long been a concern in marketing [30], but surveys by Patrick [61] as well as Hoegg and Alba [27] reveal increasing interest over the last several years, with particular focus on how aesthetics influence consumer choice. Marketing researchers are practical, with Patrick advocating for an "everyday aesthetics [that] is a diverse and dynamic experience, that more often than not leads to specific actions: cleaning, purchasing, repairing, discarding...." We focus here on a few papers particularly relevant to visualization.

Two models of persuasive communication provide meaningful context for aesthetics in visualization. Petty et al.'s elaboration likelihood model [63] (ELM, Fig. 2), has been widely applied in marketing research. It describes two paths to persuasion: a "central," deliberate path for more reasoned appeals; and a "peripheral," automatic path for more visceral appeals. In Kahneman's terminology [37], central appeals are made to the "slow" mind, while peripheral appeals are made to the "fast" mind. Appeals taking the central path require more attention, but have longer lasting persuasive impact. Appeals taking the peripheral path require less attention, but their effect is more transient. The ELM has some similarity to Leder's model of aesthetic experience [46], with both having components dominated by Kahneman's "slow" and "fast" thinking. The model of visual metaphor and conceptual complexity [81] relates the complexity of a visual comparison to viewer appreciation, positing that viewers prefer comparisons that are neither too simple, nor too challenging. This echoes the relationship between aesthetic complexity and beauty.

Two studies illustrate the importance of aesthetics in product choice. Creusen and Schoormans studied the roles that product appearance plays for consumers [17]. Participants chose their preferred answering machine from a set of three, then verbally explained their choice, with researchers coding their reasons into six categories: simple visual visual appeal; symbolic associations communicating self-image; utility, e.g. a redial button; ergonomics or usability; attention-drawing ability; and ease of categorization or prototypicality. Roughly two thirds of participants gave aesthetic reasons for their choice, while nearly half offered symbolic reasons. Reimann et al. [66] showed participants images of products with standard or aesthetic packaging, and asked them if they would buy each product. Participants chose aesthetically packaged products more often, even when they had unfamiliar branding and higher cost. Reaction times and fMRI confirmed the impact of good aesthetics.

Clearly, visual aesthetics play an important role in marketing communication. Given this, the shared cognitive roots [37] of the ELM [63] and Leder's aesthetic model [46] suggest the possibility that each aesthetic component may be most effective in aiding either lasting central or transient peripheral appeals. For example, while the right level of perceptual complexity may help capture viewer attention; the right level of conceptual complexity may help retain that attention. Yet contextual effects should not be overlooked: both Creusen and Schoormans [17] and Reimann et al. [66] remind us that a message's nature, audience and environment will change the importance of overall aesthetic appeal in a product, and the relative importance of aesthetic components.

\section{Aesthetics in Communicative Visualization}

Communicative visualization practitioners seek to use data visualization to deliver their messages clearly and persuasively, often in noisy, distracting contexts such as scientific communication [12]. Aesthetic improvements are one of the few visual options available to practitioners for reaching these goals - aside from the visualizations themselves. Kosara et al. [39] urged the use of visualizations in such a persuasive role, while Moere [53] specifically endorsed aesthetics for persuasion with ambient visualizations. Tractinsky and Meyer [76] found that presenters used more complex embellishments in their visuals when they were trying to convince.

Yet until recently, the use of aesthetic embellishments was a hotly debated question, with Tufte [80] advocating for the simplest, unimproved visualizations by maximizing the "data-ink ratio." On the other hand, designers like Holmes [31] employed embellishments widely, which he believed would engage viewers and better communicate. When Bateman et al. examined this question [5], they found that at least for his native communicative context, Holmes was largely correct: embellishments did not interfere with understanding, and improved the long-term memorability of the visualizations. Later research by Haroz et al. [22] did not precisely replicate Bateman et al.'s work, but did add nuance: at least for simpler isotype visualizations, embellishments did harm understanding.

Communicative visualization practitioners are extremely interested in the impact of their messages. Lasting impact typically requires that viewers not only understand the visualization, but also remember it. For this reason, memorability has been a focus of communicative visualization literature. In addition to the work by Bateman et al. [5], Borgo et al. [9] found that embellishments could improve short-term recall. On the other hand, like Haroz et al. [22], Kozik and Tateosian et al. found no improvement in short term recall when visualization aesthetics were improved with artistic treatments (not embellishments) [40,74]. All of these studies are relatively small in scale. Borkin et al. $[10,11]$ employed thousands of real-world visualizations to find features that improve short-term memorability. Many features were aesthetic components: novelty, complexity, familiarity and colorfulness. 
Research on the communicative impact of aesthetic visualizations outside of memorability is limited, but two projects are especially interesting. First, Haroz et al. [22] found that in a noisy visual context, viewers first examined isotype visualizations much more than they did text or bar charts. Second, Pandey et al. [59] studied the value of simple visualizations in persuasion. When viewers did not already have strong attitudes, colored bar charts were more successful at changing attitudes than uncolored textual tables.

Two trends are particularly striking here. First, while much of the visualization work we describe has aesthetic implications, little of it directly addresses the value of aesthetic visualizations (e.g., how beautiful are isotypes?). Second, few of these visualization research efforts address higher-level, more cognitive aesthetic components (see Table 1), such as familiarity, prototypicality or fluency.

\section{Design and Research Implications}

As even this brief review makes clear, there is growing interest from other fields in the applied value of aesthetics. Despite this, we find relatively little research in the applied value of aesthetics for communicative visualization. We therefore offer speculative aesthetic design guidelines to visualization practitioners, and identify important opportunities for visualization researchers.

For practitioners of communicative visualization, we begin by noting that the guidelines we offer have not been formally tested. Nevertheless, practice does not wait for research! First, recall that beauty can create engagement: early research in communicative visualisation [24] and years of research in marketing (e.g., [66]) confirms this. Second, use low-level aesthetic components (e.g. color, complexity, symmetry) to start engagement — particularly in noisy, competitive information environments - and higher-level components (e.g. familiarity, fluency, style, content) to keep it. Finally, know your audience so that you can tune each component properly: e.g. neither to simple, nor too complex.

There are many research opportunities in communicative visualization, but we list just a few here. First, we hypothesize above that low-level aesthetic components are most effective for engaging initially, especially in competitive information environments; and that high-level aesthetic components are best for lasting impact. We base these hypotheses on the shared similarities of the ELM [63] and Leder's aesthetic model [46] to Kahneman's two-system model of "fast" and "slow" thinking [37], but they should be confirmed. Second, in service of that goal, we urge application of the research methods of marketing research, which shares many goals with communicative visualization. Finally, as Table 2 shows, many measures of visualization effects on viewers are underutilized - researchers should begin using them more often.

\section{ACKNOWLEDGMENTS}

Our thanks to Radi Reza for initiating this project.

\section{References}

[1] T. R. Alley and M. R. Cunningham. Article commentary: Averaged faces are attractive, but very attractive faces are not average. Psychological science, 2(2):123-125, 1991.

[2] R. Arnheim. Art and visual perception. Univ of California Press, 1974.

[3] R. Baltissen and B.-M. Ostermann. Are the dimensions underlying aesthetic and affective judgment the same? Empirical Studies of the Arts, 16(2):97-113, 1998.

[4] J. Barnes et al. Complete works of Aristotle, volume 1: The revised Oxford translation, vol. 1. Princeton University Press, 2014.

[5] S. Bateman, R. L. Mandryk, C. Gutwin, A. Genest, D. McDine, and C. Brooks. Useful junk?: the effects of visual embellishment on comprehension and memorability of charts. In Proceedings of the SIGCHI Conference on Human Factors in Computing Systems, pp. 2573-2582. ACM, 2010.
[6] M. Bauerly and Y. Liu. Effects of symmetry and number of compositional elements on interface and design aesthetics. Intl. Journal of Human-Computer Interaction, 24(3):275-287, 2008.

[7] D. E. Berlyne. Aesthetics and psychobiology. 1971.

[8] J. Bölte, T. M. Hösker, G. Hirschfeld, and M. T. Thielsch. Electrophysiological correlates of aesthetic processing of webpages: a comparison of experts and laypersons. PeerJ, 5:e3440, 2017.

[9] R. Borgo, A. Abdul-Rahman, F. Mohamed, P. W. Grant, I. Reppa, L. Floridi, and M. Chen. An empirical study on using visual embellishments in visualization. IEEE Transactions on Visualization \& Computer Graphics, (12):2759-2768, 2012.

[10] M. A. Borkin, Z. Bylinskii, N. W. Kim, C. M. Bainbridge, C. S. Yeh, D. Borkin, H. Pfister, and A. Oliva. Beyond memorability: Visualization recognition and recall. IEEE transactions on visualization and computer graphics, 22(1):519-528, 2016.

[11] M. A. Borkin, A. A. Vo, Z. Bylinskii, P. Isola, S. Sunkavalli, A. Oliva, and $\mathrm{H}$. Pfister. What makes a visualization memorable? IEEE Trans. on Visualization and Computer Graphics, 19(12):2306-2315, 2013.

[12] R. V. Burri and J. Dumit. 13 social studies of scientific imaging and visualization. Handbook of science \& technology studies, 297, 2008.

[13] N. Cawthon and A. V. Moere. A conceptual model for evaluating aesthetic effect within the user experience of information visualization. In null, pp. 374-382. IEEE, 2006.

[14] A. Chatterjee. Neuroaesthetics: a coming of age story. Journal of cognitive neuroscience, 23(1):53-62, 2011.

[15] L. Cooper, E. Hamilton, H. Cairns, et al. The collected dialogues of Plato, vol. 18. Princeton University Press, 1961.

[16] B. Coulter, H. Delisle, C. DeMillo, J. DuFresne, and B. Paulus. Graphic design curriculum, 2021.

[17] M. E. Creusen and J. P. Schoormans. The different roles of product appearance in consumer choice. Journal of product innovation management, 22(1):63-81, 2005.

[18] Q. Dou, X. S. Zheng, T. Sun, and P.-A. Heng. Webthetics: quantifying webpage aesthetics with deep learning. International Journal of Human-Computer Studies, 124:56-66, 2019.

[19] M. Douneva, R. Jaron, and M. T. Thielsch. Effects of different website designs on first impressions, aesthetic judgements and memory performance after short presentation. Interacting with Computers, 28(4):552-567, 2016.

[20] G. T. Fechner. Vorschule der aesthetik, vol. 1. Breitkopf \& Härtel, 1876.

[21] C.-D. Ham, M. R. Nelson, and S. Das. How to measure persuasion knowledge. International Journal of Advertising, 34(1):17-53, 2015.

[22] S. Haroz, R. Kosara, and S. L. Franconeri. Isotype visualization: Working memory, performance, and engagement with pictographs. In Proceedings of the 33rd annual ACM conference on human factors in computing systems, pp. 1191-1200. ACM, 2015.

[23] D. Harper et al. Online etymology dictionary, 2001

[24] L. Harrison, K. Reinecke, and R. Chang. Infographic aesthetics: Designing for the first impression. In Proc. ACM Conf. on Human Factors in Computing Systems, pp. 1187-1190. ACM, 2015.

[25] M. Hassenzahl, M. Burmester, and F. Koller. Attrakdiff: Ein fragebogen zur messung wahrgenommener hedonischer und pragmatischer qualität. In Mensch \& Computer 2003, pp. 187-196. Springer, 2003.

[26] P. Hekkert, D. Snelders, and P. C. Van Wieringen. 'most advanced, yet acceptable': Typicality and novelty as joint predictors of aesthetic preference in industrial design. Brit. J. Psych., 94(1):111-124, 2003.

[27] J. Hoegg and J. W. Alba. A role for aesthetics in consumer psychology. In Handbook of consumer psychology, pp. 726-747. Routledge, 2018.

[28] L. Höfel and T. Jacobsen. Temporal stability and consistency of aesthetic judgments of beauty of formal graphic patterns. Perceptual and Motor Skills, 96(1):30-32, 2003.

[29] L. Höfel and T. Jacobsen. Electrophysiological indices of processing aesthetics: Spontaneous or intentional processes? International Journal of Psychophysiology, 65(1):20-31, 2007.

[30] M. B. Holbrook. Some preliminary notes on research in consumer esthetics. ACR North American Advances, 1980.

[31] N. Holmes. Designer's guide to creating charts \& diagrams. WatsonGuptill Publications New York, 1984.

[32] J. Huh, M. S. Ackerman, and R. Douglas. The use of aesthetics in hci 
systems. In CHI'07 extended abstracts on Human factors in computing systems, pp. 2441-2446, 2007.

[33] D. Hume. "of the standard of taste," essays moral and political. 1894.

[34] T. Jacobsen. Beauty and the brain: culture, history and individual differences in aesthetic appreciation. J. Anat., 216(2):184-191, 2010.

[35] T. Jacobsen and L. Höfel. Aesthetic judgments of novel graphic patterns: analyses of individual judgments. Perceptual and motor skills, 95(3):755-766, 2002.

[36] B. Jaworski, P. Drucker, R. Lutz, G. Marshall, L. Price, and R. Varadarajan. What is marketing?, 2017.

[37] D. Kahneman and P. Egan. Thinking, fast and slow, vol. 1. Farrar, Straus and Giroux New York, 2011.

[38] I. Kant. Critique of judgement (jh bernard, trans., 1914), 1790.

[39] R. Kosara. Presentation-oriented visualization techniques. IEEE computer graphics and applications, 36(1):80-85, 2016.

[40] P. Kozik. Performance of impressionist visualizations on measures of recognition and trend identification. $\mathrm{PhD}$ thesis, U. Brit. Colmb., 2015

[41] C.-Y. Lai, P.-H. Chen, S.-W. Shih, Y. Liu, and J.-S. Hong. Computational models and experimental investigations of effects of balance and symmetry on the aesthetics of text-overlaid images. International journal of human-computer studies, 68(1-2):41-56, 2010.

[42] J. H. Langlois, L. Kalakanis, A. J. Rubenstein, A. Larson, M. Hallam, and M. Smoot. Maxims or myths of beauty? a meta-analytic and theoretical review. Psychological bulletin, 126(3):390, 2000.

[43] J. H. Langlois, J. M. Ritter, L. A. Roggman, and L. S. Vaughn. Facial diversity and infant preferences for attractive faces. Developmental Psychology, 27(1):79, 1991.

[44] T. Lavie and N. Tractinsky. Assessing dimensions of perceived visual aesthetics of web sites. International journal of human-computer studies, 60(3):269-298, 2004.

[45] H. Leder, B. Belke, A. Oeberst, and D. Augustin. A model of aesthetic appreciation and aesthetic judgments. British journal of psychology, 95(4):489-508, 2004.

[46] H. Leder and M. Nadal. Ten years of a model of aesthetic appreciation and aesthetic judgments: the aesthetic episode-developments \& challenges in empirical aesthetics. Br. J. Psych., 105(4):443-464, 2014.

[47] G. Lindgaard, C. Dudek, D. Sen, L. Sumegi, and P. Noonan. An exploration of relations between visual appeal, trustworthiness and perceived usability of homepages. ACM Transactions on ComputerHuman Interaction (TOCHI), 18(1):1, 2011

[48] G. Lindgaard, G. Fernandes, C. Dudek, and J. Brown. Attention web designers: You have 50 milliseconds to make a good first impression! Behaviour \& information technology, 25(2):115-126, 2006.

[49] C. Martindale. The pleasures of thought: A theory of cognitive hedonics. The Journal of Mind and Behavior, pp. 49-80, 1984.

[50] C. Martindale, K. Moore, and J. Borkum. Aesthetic preference: Anomalous findings for berlyne's psychobiological theory. The American Journal of Psychology, pp. 53-80, 1990

[51] Merriam-Webster. Webster's 9th new collegiate dictionary. 1983.

[52] E. Michailidou, S. Harper, and S. Bechhofer. Visual complexity and aesthetic perception of web pages. In Proc. ACM Conf. on Design of Communication, pp. 215-224, 2008

[53] A. V. Moere. Towards designing persuasive ambient visualization. In Issues in the Design \& Evaluation of Ambient Information Systems Workshop, pp. 48-52, 2007.

[54] G. E. Moore. Principia Ethica:(1903), vol. 960. Cambr. U. Pr., 1959

[55] M. Moshagen and M. T. Thielsch. Facets of visual aesthetics. International journal of human-computer studies, 68(10):689-709, 2010.

[56] D. A. Norman. Emotional design: Why we love (or hate) everyday things. Basic Civitas Books, 2004.

[57] S. E. Palmer and K. B. Schloss. An ecological valence theory of human color preference. Proc. Nat. Acad. Sciences, p. 200906172, 2010.

[58] S. E. Palmer, K. B. Schloss, and J. Sammartino. Visual aesthetics and human preference. Annual review of psychology, 64:77-107, 2013.

[59] A. V. Pandey, A. Manivannan, O. Nov, M. Satterthwaite, and E. Bertini. The persuasive power of data visualization. IEEE transactions on visualization and computer graphics, 20(12):2211-2220, 2014.

[60] S.-e. Park, D. Choi, and J. Kim. Visualizing e-brand personality: Exploratory studies on visual attributes and e-brand personalities in korea. Int. Journal of Human-Computer Interaction, 19(1):7-34, 2005.
[61] V. M. Patrick. Everyday consumer aesthetics. Current Opinion in Psychology, 10:60-64, 2016.

[62] R. E. Petty and J. T. Cacioppo. The elaboration likelihood model of persuasion. In Communication \& Persuasion, pp. 1-24. Springer, 1986.

[63] R. E. Petty, J. T. Cacioppo, and D. Schumann. Central and peripheral routes to advertising effectiveness: The moderating role of involvement. Journal of consumer research, 10(2):135-146, 1983.

[64] B. Pham. Design for aesthetics: interactions of design variables and aesthetic properties. In Human Vision and Electronic Imaging IV, vol. 3644, pp. 364-371. Int. Society for Optics \& Photonics, 1999.

[65] V. S. Ramachandran and W. Hirstein. The science of art: A neurological theory of aesthetic experience. J. Conscns. Studies, 6(6-7):15-51, 1999.

[66] M. Reimann, J. Zaichkowsky, C. Neuhaus, T. Bender, and B. Weber. Aesthetic package design: A behavioral, neural, and psychological investigation. Journal of Consumer Psychology, 20(4):431-441, 2010.

[67] K. Reinecke, T. Yeh, L. Miratrix, R. Mardiko, Y. Zhao, J. Liu, and K. Z. Gajos. Predicting users' first impressions of website aesthetics with a quantification of perceived visual complexity and colorfulness. In Proceedings of the SIGCHI Conference on Human Factors in Computing Systems, pp. 2049-2058. ACM, 2013.

[68] I. Robinson and M. Robinson. Measures of emotion: a review. Cogn. Emot, 23:209-237, 2009.

[69] S. Sagmeister and J. Walsh. Beauty. London : Phaidon Press Limited ; New York, New York : Phaidon Press Inc., 2018., London, 2018.

[70] C. Sartwell. Six names of beauty. Routledge, 2013.

[71] C. Sartwell. Beauty. In E. N. Zalta, ed., The Stanford Encyclopedia of Philosophy. Metaphysics Rch. Lab, Stanford Univ., winter ed., 2017.

[72] K. B. Schloss and S. E. Palmer. Aesthetic response to color combinations: preference, harmony, and similarity. Attention, Perception, \& Psychophysics, 73(2):551-571, 2011.

[73] J. G. Snodgrass and M. Vanderwart. A standardized set of 260 pictures: norms for name agreement, image agreement, familiarity, and visual complexity. J. Exp. Psych.: Human Lrng. \& Mmry., 6(2):174, 1980.

[74] L. G. Tateosian, C. G. Healey, and J. T. Enns. Engaging viewers through nonphotorealistic visualizations. In Proc. Symp. Non-Photorealistic Animation \& Rendering, pp. 93-102. ACM, 2007.

[75] N. Tractinsky. Visual aesthetics. The Encyclopedia of HumanComputer Interaction, 2nd Ed., 2013.

[76] N. Tractinsky and J. Meyer. Chartjunk or goldgraph? effects of presentation objectives and content desirability on information presentation. MIS quarterly, pp. 397-420, 1999.

[77] A. N. Tuch, J. A. Bargas-Avila, and K. Opwis. Symmetry and aesthetics in website design: It'sa man's business. Computers in Human Behavior, 26(6):1831-1837, 2010.

[78] A. N. Tuch, J. A. Bargas-Avila, K. Opwis, and F. H. Wilhelm. Visual complexity of websites: Effects on users' experience, physiology, performance, and memory. International journal of human-computer studies, 67(9):703-715, 2009.

[79] A. N. Tuch, E. E. Presslaber, M. Stöcklin, K. Opwis, and J. A. BargasAvila. The role of visual complexity and prototypicality regarding first impression of websites: Working towards understanding aesthetic judgments. Int. J. Human-Computer Studies, 70(11):794-811, 2012.

[80] E. R. Tufte. The visual display of quantitative information. 1983.

[81] M. Van Mulken, A. van Hooft, and U. Nederstigt. Finding the tipping point: Visual metaphor and conceptual complexity in advertising. Journal of Advertising, 43(4):333-343, 2014

[82] P. Vitruvius, V. Rose, and H. Müller-Strübing. De architectura libri decem. Teubneri, 1867

[83] A. W. White. The elements of graphic design: space, unity, page architecture, and type. Skyhorse Publishing, Inc., 2011.

[84] O. Wu, Y. Chen, B. Li, and W. Hu. Evaluating the visual quality of web pages using a computational aesthetic approach. In Proceedings of the fourth ACM international conference on Web search and data mining, pp. 337-346, 2011.

[85] R. B. Zajonc. Feeling and thinking: Preferences need no inferences. American psychologist, 35(2):151, 1980.

[86] J. Zhang, J. Yu, K. Zhang, X. S. Zheng, and J. Zhang. Computational aesthetic evaluation of logos. ACM Trn. Apd. Pcp., 14(3):1-21, 2017. 